\title{
What are the active ingredients of mass media and social marketing interventions to increase HIV testing amongst MSM
}

\author{
Julie Riddell' ${ }^{1}$ Lisa McDaid ${ }^{1}$, Nicola Boydell², Gemma Teal ${ }^{3}$, Nicky Coia ${ }^{4}$, Paul Flowers ${ }^{5}$ \\ ${ }^{1} \mathrm{MRC} / \mathrm{CSO}$ Social and Public Health Sciences Unit, University of Glasgow, Glasgow, Scotland, UK; ${ }^{2}$ Usher Institute of Population Health Sciences and \\ Informatics, University of Edinburgh, Edinburgh, Scotland; ${ }^{3}$ The Glasgow School of Art, Glasgow, Scotland, UK; ${ }^{4} \mathrm{NHS}$ Greater Glasgow \& Clyde, Glasgow, \\ Scotland, UK; ${ }^{5}$ Glasgow Caledonian University, Glasgow, Scotland, UK
}

\section{Introduction}

Increasing HIV testing among Men who have sex with men (MSM) is foundational to wider biomedical HIV prevention. Mass media interventions targeting MSM are effective, however there is little understanding of the specific active ingredients of such interventions.

Through a systematic review we conducted secondary analyses of existing interventions to identify their specific active content.

\section{Methods}

Five electronic databases searched using detailed search strategies and standard MESH terms for HIV, MSM and social marketing/mass media interventions. Structured data extraction tools were used.

\section{Assessing mechanisms of action (theory and theoretical constructs) The first 11 items of the Theory coding scheme (TCS) were used to explore relationships between theory, target behaviours and their role in information development and evaluation. Theoretical content of interventions were examined using 14 domains of the Theoretical Domains Framework (TDF)}

\section{Behaviour Change Taxonomy}

Behaviour Change Techniques (BCTs) within the BCTTV1 were coded as implicit or explicit within each intervention descriptions and/or materials (e.g. posters).

\section{Effectiveness}

Studies were assessed and categorized in terms of relative effectiveness (i.e negative effect, no effect, an effect on the antecedent of behaviour, some positive desired behaviour change and clear behaviour change in desired direction).

\section{Analysis}

TDF domains, individual and grouped BCTs were calculated across the interventions to enable the description of 'standard' content. These same elements were then mapped against the categories of effectiveness to enable the identification of unique components present only within the most effective interventions.

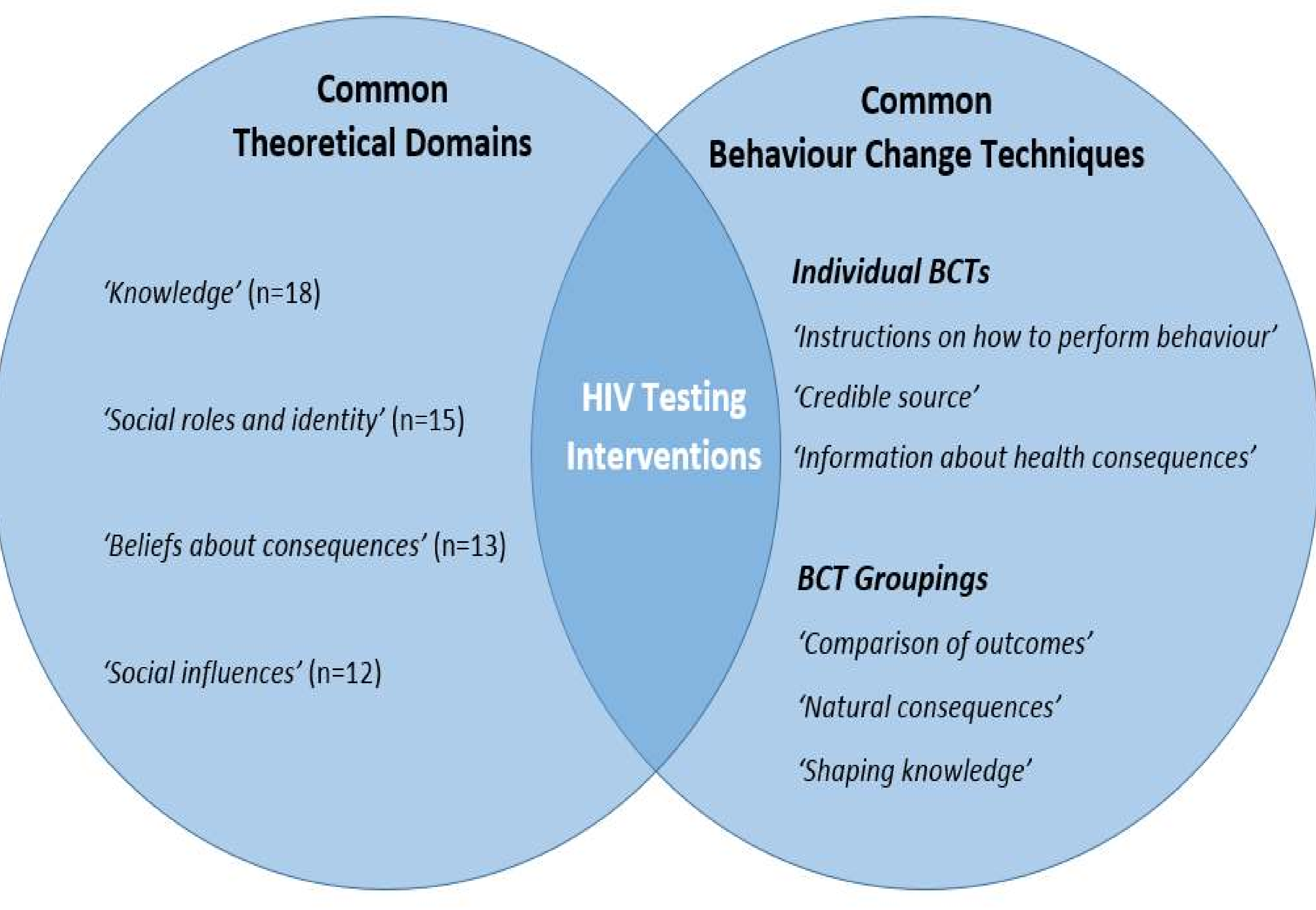

Figure 1: Common BCTs and Theory domains

\section{Results}

19 studies met the inclusion criteria,

\section{Theory and Theoretical Constructs}

Only five studies reported an explicit theoretical basis to their intervention

Most commonly used domains were as follows (figure 1):

- 'Knowledge'

- 'Social roles and identity'

- 'Beliefs about consequences'

- 'Social influences'

\section{Behaviour Change Techniques}

Evidence of coherence between the TDF mechanisms of action and the BCT mechanisms of change identified within the included interventions.

The most common connection was between the TDF domain of 'Knowledge' and the individual BCTs relating to 'Instruction on how to perform a behaviour' and 'Information about health consequences'.

Three BCT groupings and three individual BCTs formed the backbone of most interventions and can be considered standard content (figure 1).

\section{Patterns of Effectiveness}

We identified patterns of BCTs and mechanisms of action that when combined with standard content (figure 1) could contribute to developing the optimal HIV testing intervention (figure 2).

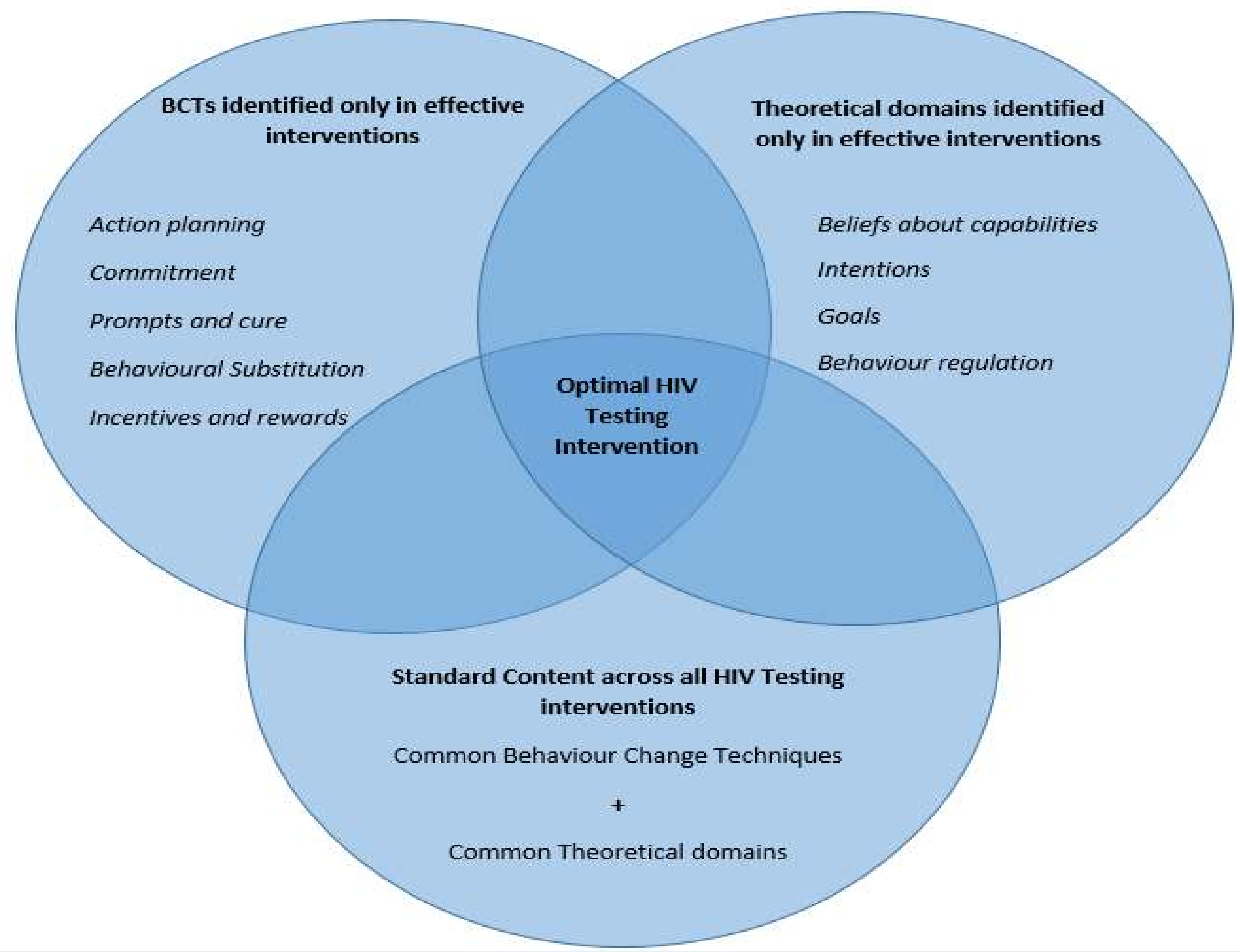

Figure 2: Standard content and effective BCTS/ theoretical domains

\section{Discussion}

Our novel and rigorous approach has identified key mechanisms of behaviour change that can be utilised to boost or reinforce standard intervention content when developing evidence-based social marketing interventions to promote regular HIV testing.

Finally, a clear and detailed report of the intervention development process is key to furthering our understanding of what works, why and for whom.

\section{Acknowledgements}

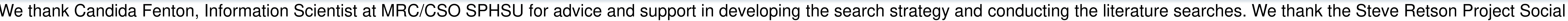
Marketing Campaign Advisory Group for their support and advice in the development of this project and Andry Cruz Diaz for translation services.

Funding :

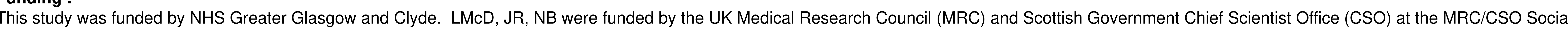
\& Public Health Sciences Unit (SPHSU), University of Glasgow (MC_UU_12017/11, SPHSU11). 